\title{
Extraskeletal myxoid chondrosarcoma arising from the clavicle
}

\author{
SHIGETO NAKAZORA ${ }^{1}$, KATSUYUKI KUSUZAKI ${ }^{1}$, TAKAO MATSUBARA ${ }^{1}$, KEN SHINTANI $^{1}$, \\ AKIHIKO MATSUMINE ${ }^{1}$, KAZUO FUKUTOME ${ }^{2}$ and ATSUMASA UCHIDA ${ }^{1}$ \\ ${ }^{1}$ Department of Orthopedic Surgery and ${ }^{2}$ Second Department of Pathology, \\ Mie University Faculty of Medicine, Edobashi, Tsu City, Mie 514-8507, Japan
}

Received January 3, 2006; Accepted March 1, 2006

\begin{abstract}
This report presents an extremely rare case of extraskeletal myxoid chondrosarcoma (EMC) arising from the clavicular periosteum. To the best of our knowledge, this may be the first detailed report of its clinicopathological findings. The patient was a 48-year-old man. Plain radiography and CT did not demonstrate any osteolytic lesion or periosteal reaction in the right clavicle. However, MRI showed an isosignal-intensity mass on T1-weighted images and a homogeneous high signal intensity lesion on T2-weighted images. The histological findings of the widely resected tumor were consistent with the diagnosis of extraskeletal myxoid chondrosarcoma. Preoperative diagnosis of extraskeletal myxoid chondrosarcoma at an unusual location, as in this case, is difficult not only with imaging examinations alone, but sometimes even after histological examination of biopsy specimens.
\end{abstract}

\section{Introduction}

Extraskeletal myxoid chondrosarcoma is a rare soft tissue sarcoma. We encountered a case of extraskeletal myxoid chondrosarcoma arising from the clavicular periostenum. A thorough search of the literature conducted to the best of our ability revealed that there has never been a case report on clavicular extraskeletal myxoid chondrosarcoma arising specifically from the periostenum. In this study, we present a detailed report of the clinicopathologic features of this rare case.

\section{Case report}

The patient, a 48-year-old man who first noticed a swelling over the right clavicular bone in 1996, visited our hospital in April 2001 because the swelling appeared to be gradually

Correspondence to: Dr K. Kusuzaki, Department of Orthopedic Surgery, Mie University Faculty of Medicine, Edobashi, Tsu City, Mie 514-8507, Japan

E-mail: kusu@clin.medic.mie-u.ac.jp

Key words: extraskeletal myxoid chondrosarcoma, periostenum, clavicle, histological diagnosis, fusion gene increasing in size. He had no history of any major illness, such as cancer, trauma, or a hereditary disease.

Upon examination, a tender mass with an elastic-hard consistency measuring $4.2 \times 5.0 \mathrm{~cm}$ in size, with overlying erythema and edema of the skin, warm to the touch, was palpable above the shaft of the right clavicle (Fig. 1). The tumor was well demarcated from the surrounding structures and showed poor mobility. Movements of the right shoulder induced slight pain around the clavicle. Plain radiographic examination did not reveal an osteolytic lesion, or cortical thinning or expansion of the right clavicle; no calcification or periosteal reaction was detectable (Fig. 2). CT examination also revealed no osteolytic lesion, or thinning or expansion of the clavicular cortex (Fig. 3). Sagittal MR images of the right clavicle revealed a very close relation between the mass and cortex of the clavicle, and T1-weighted images revealed a homogeneous isosignal-intensity mass arising from the right clavicle (Fig. 4A). On T2-weighted imaging, this lesion was visualized as a homogeneous high-intensity signal at the center (Fig. 4B). The high signal intensity lesion was enhanced on T1-weighted images obtained after intravenous injection of gadolinium-DTPA (Fig. 4C). Thallium ${ }^{201}$ scintigraphy demonstrated strong accumulation of RI in the lesion, whereas no such accumulation was observed on Tc ${ }^{99 m}$-HMDP scintigraphy. These findings, obtained by various imaging examinations, suggested the diagnosis of a malignant tumor, such as a synovial sarcoma, periosteal osteosarcoma or slowgrowing metastatic bone tumor. Needle biopsy was therefore performed before surgical resection. Because histopathological examination of the biopsy specimens suggested synovial sarcoma or chondrosarcoma, and the potential functional deficit after surgery was considered to be minimal, a wide tumor resection that included the clavicle and its surrounding muscles was performed. At 36 months after surgery, there was no clinical evidence of local tumor recurrence, and the patient could fully move his shoulder joint without pain and only mild cosmetic complaints.

A longitudinal section of the widely resected tumor revealed a white or yellow-white surface, and the tumor contained a hematoma. A close relation was observed between the tumor periphery and clavicular cortex, confirming the MRI findings. Since the mass was strongly attached to the cortical bone of the clavicle and there was no lesion in the bone marrow, it was surmised that the tumor arose from the peritoneum of the clavicle (Fig. 5). 


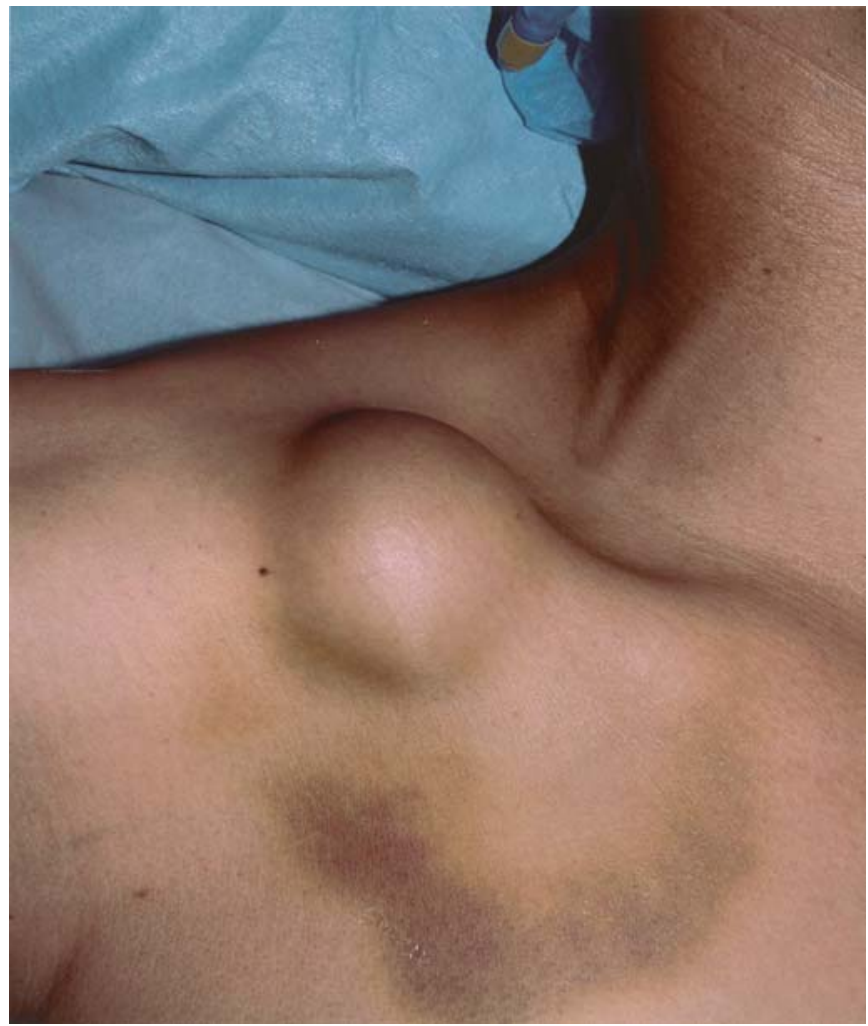

Figure 1. The mass with an elastic-hard consistency is seen over the shaft of the right clavicle, measuring $4.2 \times 5.0 \mathrm{~cm}$ in size.

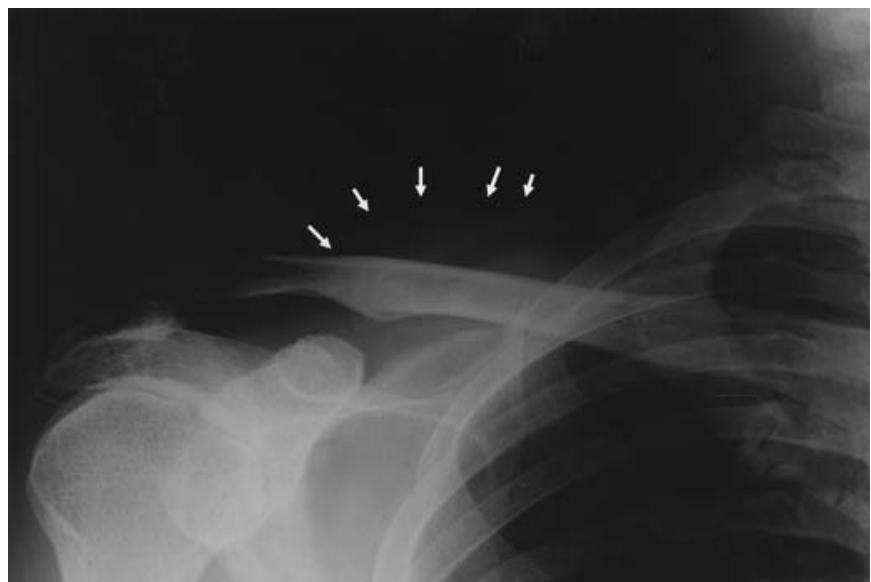

Figure 2. A plain radiograph shows a soft-tissue mass over the clavicle (arrows), but no osteolytic lesion or cortical expansion of the right clavicle. No calcification or periosteal reaction was observed.

Microscopically, the tumor exhibited three structural patterns: a part with dense striform arrangement of spindleshaped cells with atypical nuclei (Fig. 6A and B); a part with an abundant and relatively loose myxomatous framework (Fig. 6C); and a part showing a hemorrhage and necrosis (Fig. 6D). Immunohistochemically, the tumor was positive for vimentin and S-100 protein, while negative results were obtained for pan-cytokeratin, epithelial membrane antigen, $\alpha$-smooth muscle actin, HHF35, and MIC2.

In a reverse-transcription polymerase chain reaction (RT-PCR) assay, a characteristic EWS-CHN/TEC fusion

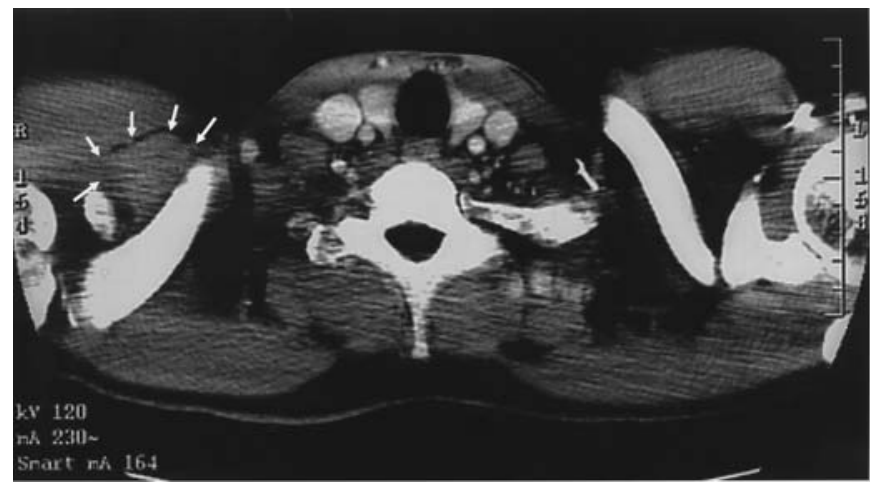

Figure 3. CT scans showing a soft-tissue mass over the right clavicle (arrows), but no osteolytic lesion, or thinning or expansion of the cortex.

A

B
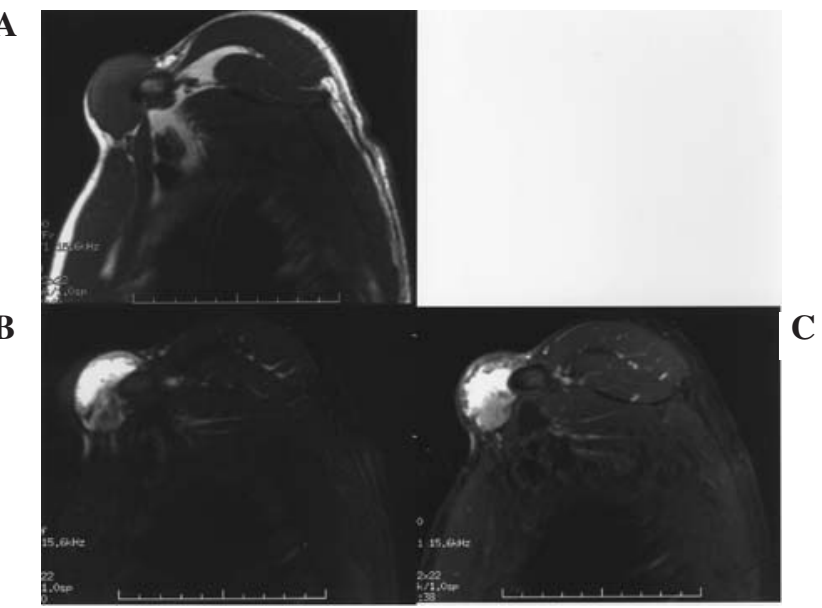

Figure 4. (A) An MRI showing a homogeneous isosignal-intensity mass on T1-weighted images of the right clavicle. (B) On T2-weighted images, the mass is visualized as a homogeneous high-intensity signal at the center. (C) Intravenous injection of gadolinium-DTPA enhanced the high signal intensity lesion on $\mathrm{T} 2$-weighted images.

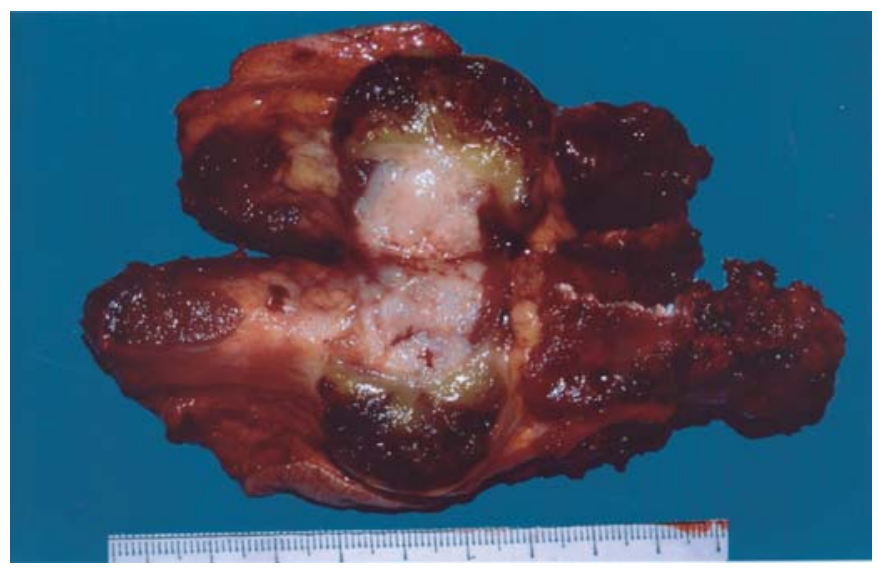

Figure 5. The surface of the tumor after longitudinal sectioning shows a white or yellow-white matrix containing a hematoma. A close relation between the periphery of the tumor and the clavicle was detected.

gene transcript was detected, although the results for expression of SYT-SSX 1 and 2 were negative. These findings were considered to be consistent with the diagnosis of extraskeletal myxoid chondrosarcoma. 
A

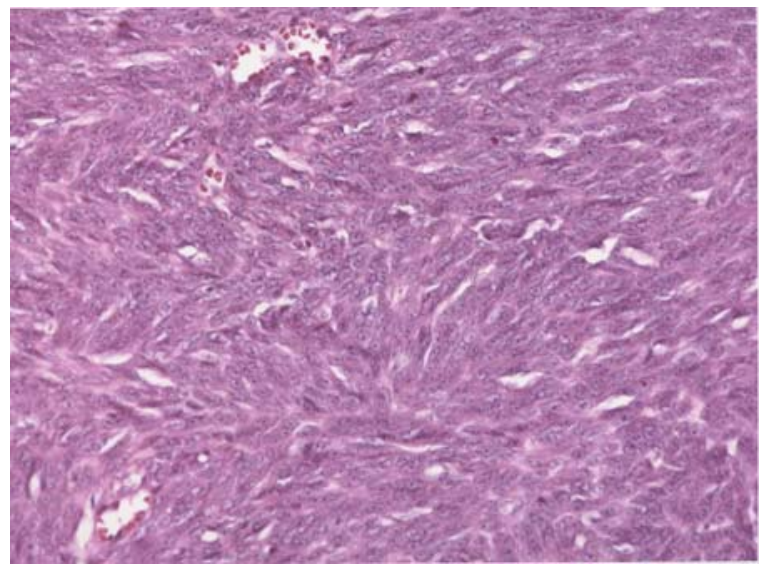

C

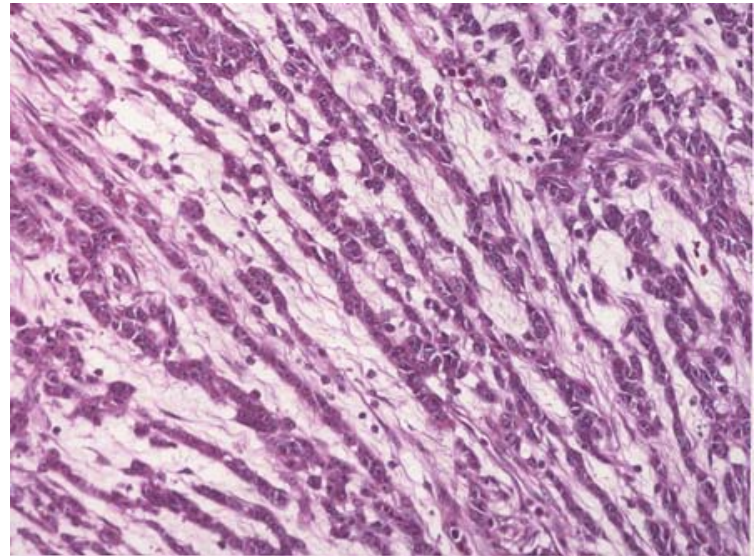

B

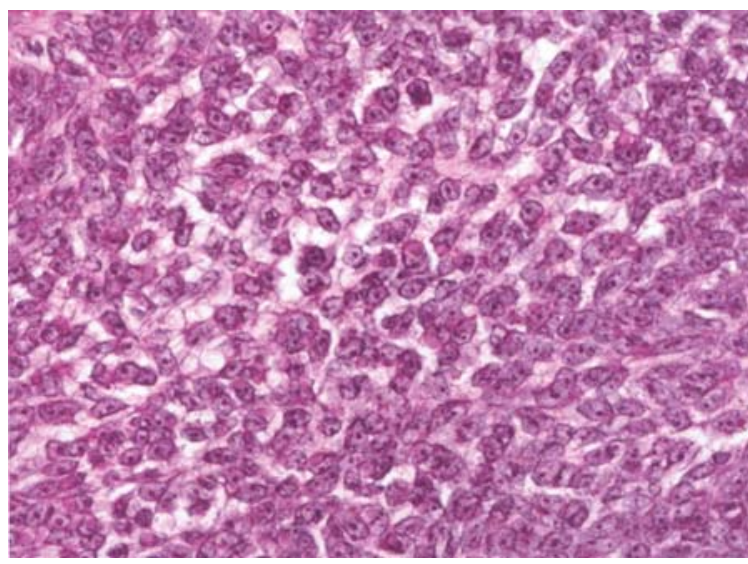

D

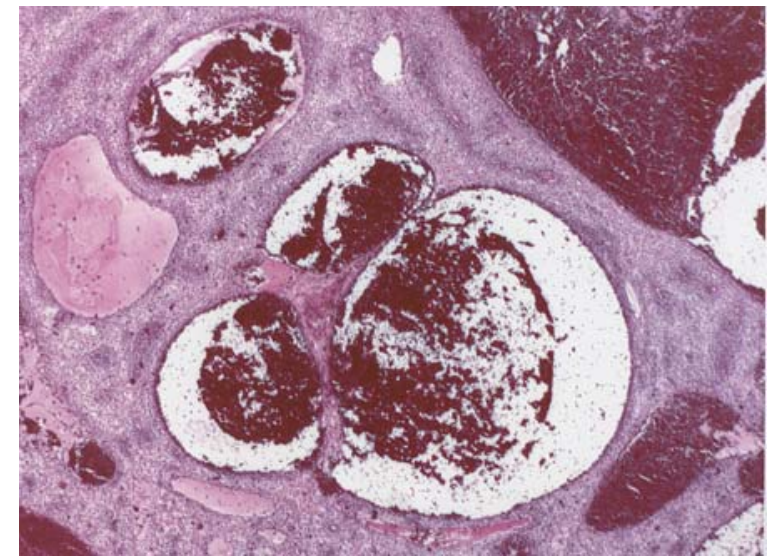

Figure 6. High-power microscopic findings of the tumor showing three structural patterns: a part with dense striform arrangement of spindle-shaped cells with atypical nuclei (A and B), with an abundant and relatively loose myxomatous framework (C), and with a hemorrhage and necrosis (D). H\&E staining.

\section{Discussion}

Extraskeletal myxoid chondrosarcoma (EMC) is a rare softtissue sarcoma with distinctive histological features. EMC was first described as a distinct clinicopathological entity by Enzinger and Shiraki in 1972 (1). Tsuneyosi et al reported that the tumor occurred at an incidence of $2.3 \%$ among 603 softtissue tumors (2). The tumor shows a predilection for persons over the age of 35 years, and has been reported to occur in both sexes, with some series reporting a male preponderance (3). Most tumors primarily occur in the deep soft tissues of the proximal extremities and limb girdles, especially the musculature, although occasional tumors have been reported as confined to subcutaneous tissue (4). Rare tumors have been described in unusual locations, including the mediastinum, retroperitoneum, abdomen, and the digits (5-7). To the best of our knowledge, there has been no case report on a tumor arising from the periosteum, clavicle or any other bone.

On plain radiography and CT, EMC is visualized as a mass with soft-tissue density and no distinctive radiological features; calcification is usually absent or not prominent if present. In our case, no calcification was detected.

The mass was visualized as an isosignal-intensity mass on T1-weighted MR images and as a high-signal-intensity mass on T2-weighted MR images, probably explained by the abundant myxoid content of the tumor. Hemorrhagic changes may sometimes be recognized on T1-weighted MR images as areas of high signal intensity. In the present case also, signal intensities consistent with myxoid content of the tumor and a hemorrhage were observed.

EMC has a distinctive histologic appearance and is characterized by lobular growth of oval or spindle-shaped acidophilic cells. The lobules are arranged in cords or strands in a prominent myxoid stroma. In a sectioned portion, the tumor exhibited a gelatinous gray to tan-brown surface, depending on the amount of hemorrhage, a frequent feature of the tumor (8). In the present case, a dense arrangement of short spindle-shaped or ovoid-shaped cells rich in cytoplasm was visualized, and the typical lobular pattern with a chondroid stroma was not prominent.

Immunohistochemically, EMC shows variable expression of vimentin, S-100, and epithelial membrane antigen, which are of little help in the diagnosis. Although S-100 was once regarded as a consistent immunohistochemical marker, a study indicated that most EMCs are S-100-negative and many of the S-100-positive cases also show only focal staining (9). Our case showed positivity for only the S-100 protein.

Our differential diagnoses included other soft-tissue tumors with a myxoid component, such as extraskeletal chondroma, a myxoid variant of MFH, myxoid liposarcoma, and juxtacortical chondrosarcoma, and soft-tissue tumors with densely arranged short spindle-shaped or ovoid cells, such as synovial sarcoma or Ewing/PNET tumor.

Cytogenetically, EMC is characterized by a $\mathrm{t}(9 ; 22)$ (q22;q12) reciprocal chromosomal translocation resulting in the expression of the EWS-CHN/TEC fusion gene in a 
majority of cases $(3,10)$. Two variant translocations, $\mathrm{t}(9 ; 17)$ $(\mathrm{q} 22 ; \mathrm{q} 11)$ and $\mathrm{t}(9 ; 15)(\mathrm{q} 22 ; \mathrm{q} 21)$, resulting in expression of the TAF2N-CHN/TEC or TCF12-CHN/TEC fusion gene, respectively, have also been documented in EMC $(11,12)$. Because such fusion genes are specific to EMC, molecular detection of these characteristic fusion genes could serve as a useful diagnostic adjunct for the final diagnosis of EMC, particularly in cases with atypical clinicopathological features. In our case, we detected the EWS-CHN fusion gene, but not SYT-SSX 1 or 2 in the tumor. Therefore, we finally arrived at the diagnosis of EMC, even though a cortical origin of the tumor, especially at the clavicle, has never been reported.

Although EMC appears to exhibit a tendency towards a high rate of local recurrence and distant metastasis, predominantly to the lung, sometimes years after the initial diagnosis, tumor-related death often occurs only after a long survival period (8). According to a study by Meis-Kindblom et al, the estimated 5-, 10-, and 15-year survival rates were $90 \%, 70 \%$, and $60 \%$, respectively (4). Therefore, EMC should be considered an intermediate-grade rather than a low-grade malignant neoplasm.

The treatment of patients with localized EMC should include excision of the primary tumor with a wide surgical margin. If a wide margin cannot be obtained, a high rate of local recurrence is observed with poor response to chemotherapy and radiotherapy. Therefore, surgery continues to be the treatment of choice, both for primary EMC and recurrent or metastatic tumors. Our case also showed a favorable clinical course after surgery.

In conclusion, we encountered a rare case of EMC arising from the clavicular periostium. For the final diagnosis, expression of the fusion gene, EWS-CHN, was more useful than the histological features.

\section{Acknowledgements}

This work was conducted at the Mie University Faculty of Medicine.

\section{References}

1. Enzinger FM and Shiraki M: Extraskeletal myxoid chondrosarcoma: an analysis of 34 cases. Hum Pathol 3: 421-435, 1972.

2. Tsuneyoshi M, Enjoji M, Iwasaki H and Shirahama N: Extraskeletal myxoid chondrosarcoma: a clinicopathologic and electron microscopic study. Acta Pathol Jpn 31: 201-208, 1981.

3. Antonescu CR, Argani P, Erlandson RA, et al: Skeketal and extraskeletal myxoid chondrosarcoma: a comparative clinicopathologic, ultrastructural, and molecular study. Cancer 83: 1504-1521, 1998.

4. Meis-Kindblom JM, Bergh P, Gunterberg B, et al: Extraskeletal myxoid chondrosarcoma: a reappraisal of its morphologic spectrum and prognostic factors based on 117 cases. Am J Surg Pathol 23: 636-650, 1999.

5. Oliveira AM, Sebo TJ, McGrory JE, et al: Extraskeletal myxoid chondrosarcoma; A clinocopathologic, immunohistochemical, and ploidy analysis of 23 cases. Mod Pathol 13: 900-908, 2000.

6. Patel SR, Burgess MA, Papadopculos NE, Linke KA and Benjamin RS: Extraskeletal myxoid chondrosarcoma: long-term experience with chemotherapy. Am J Clin Oncol 18: 161-163, 1995.

7. Okamoto S, Hara K, Sumita S, et al: Extraskeletal myxoid chondrosarcoma arising in the finger. Skeletal Radiol 31: 296-300, 2002.

8. Weiss SW and Goldblum JR: Extraskeletal myxoid chondrosarcoma. In: Soft Tissue Tumors. 4th edition. Mosby, St. Louis, pp1368-1379, 2001.

9. Deitos AP, Wadden C and Fletcher CDM: Extraskeletal myxoid chondrosarcoma: an immunohistochemical reappraisal of 39 cases. Appl Immunohistochem 5: 73-77, 1997.

10. Okamoto S, Hisaoka M, Ishida T, et al: Extraskeletal myxoid chondrosarcoma: a clinicopathologic, immunohistochemical, and molecular analysis of 18 cases. Hum Pathol 32: 1116-1124, 2001.

11. Sjogren H, Meis-Kindblom JM, Kindblom LG, Hermann G, et al: Fusion of the EWS-related gene TAF2N to TEC in extraskeletal myxoid chondrosarcoma. Cancer Res 59: 5064-5067, 1999.

12. Sjogren H, Wedell B, Meis-Kindblom JM, Kindblom LG and Stenman G: Fusion of the NH2-terminal domain of the basic helix-loop-helix protein TCF12 to TEC in extraskeletal myxoid chondrosarcoma with translocation $\mathrm{t}(9 ; 15)(\mathrm{q} 22 ; \mathrm{q} 21)$. Cancer Res 60: 6832-6835, 2000. 